\title{
von Hippel-Lindau Disease with Multi-organ Cysts and Ticking Pheochromocytoma: A Rare Case
}

\author{
Smitha S Rao ${ }^{1}$, Jabamalai Ferdinant ${ }^{2}$, Puzhakkal Shikhil ${ }^{3}$, Althaf Ahmed ${ }^{4}$, Anjali Sathya ${ }^{5}$, Dhalapathy Sadacharan ${ }^{6}$
}

\begin{abstract}
Objective: A rare disease.

Background: von Hippel-Lindau (VHL) disease is an autosomal dominant familial syndrome with a multitude of benign and malignant multivisceral tumors and one-third of them harbor pheochromocytoma (PCC). We intend to present one such rare case and challenges in its surgical management.

Case: A 38-year-old lady presented with hypertension and episodic headache. Biochemical evaluation was suggestive of hypercatecholaminism. Magnetic resonance imaging (MRI) showed the presence of a heterogeneous intensity lesion in the left suprarenal area suggestive of PCC. Multiple cysts were seen in both the kidneys as well as in the pancreatic head and tail. Imaging of the brain and spine showed a left cerebellar and spinal cord hemangioblastoma, respectively. I-131 metaiodobenzylguanidine (MIBG) showed high uptake in the left suprarenal region. After adequate preparation, she underwent left adrenalectomy. Histopathology was consistent with adrenal PCC and the patient was biochemically cured of hypercatecholaminism. The presence of PCC and other manifestations of VHL type $2 \mathrm{~A}$ were evident in this patient.

Conclusion: The presence of multiple renal and pancreatic cysts can camouflage adrenal tumors intraoperatively. Careful identification, dissection, and differentiation of PCC from these cysts are crucial. Multicentricity and multifocality of PCC are not uncommon in VHL and these patients need lifelong close follow-up for other tumors as well.

Keywords: Hemangioblastoma, Pheochromocytoma, Renal and pancreatic cysts, von Hippel-Lindau disease.

World Journal of Endocrine Surgery (2019): 10.5005/jp-journals-10002-1248
\end{abstract}

\section{BACKGROUND}

von Hippel-Lindau (VHL) disease is an autosomal dominant familial syndrome with a rare incidence of $2-3$ cases per 100,000 population. Multi-visceral tumors are seen in approximately $65-75 \%$ of patients with VHL. ${ }^{1}$ It is a cancer-predisposition syndrome with a mutation in the VHL tumor-suppression gene on chromosome 3p25-26. Cysts and tumors seen due to this mutation include pancreatic neuroendocrine tumors, pancreatic cysts (35-70\%), cerebellar and spinal hemangioblastomas (60-80\%), clear cell renal cell carcinoma (RCC) in $24-45 \%$ of patients, ovarian cysts, and PCC. Most patients with VHL have positive family history. However, a de novo mutation is seen in about $20 \%$ of patients. The mean age at diagnosis is in the 20s, with high morbidity and mortality. ${ }^{2}$

$\mathrm{VHL}$ is a multisystemic syndrome characterized by benign or malignant tumors. The clinical classification of VHL comprises of three types. Type 1 is characterized by a high risk of RCC and a low risk of PCC, type $2 \mathrm{~A}$ with a high risk of hemangioblastoma and PCC with a low risk of RCC and type $2 B$ with high risk of RCC. Type $2 \mathrm{C}$ is characterized by a high risk of PCC and type 3 with Chuvash polycythemia. ${ }^{3}$

$\mathrm{VHL}$ disease can be diagnosed by the presence of any of the tumors (renal tumors, pancreatic cysts or tumors, PCC, and papillary cystadenomas of the epididymis) with a positive family history. Sporadic VHL (20\%) is diagnosed by the presence of two VHL tumors, in the absence of a positive family history or a single retinal or central nervous system (CNS) hemangioblastoma with another visceral manifestation. ${ }^{4,5}$ Germline VHL mutation identification has been reported to be a reliable method for the confirmation of diagnosis. ${ }^{6}$ The disease shows $100 \%$ penetrance by the age of 60 years, with variable expression. ${ }^{7}$ We report a 38 -year-old female diagnosed with VHL disease, presenting with a left adrenal PCC.

\author{
1-4,6 Department of Endocrine Surgery, Madras Medical College, \\ Chennai, Tamil Nadu, India \\ ${ }^{5}$ Department of Medical Endocrinology, Vijaya Group of Hospitals, \\ Chennai, Tamil Nadu, India
}

Corresponding Author: Dhalapathy Sadacharan, Department of Endocrine Surgery, Madras Medical College, Chennai, Tamil Nadu, India, Phone: +91 9790719570, e-mail: drsdhalapathy@gmail.com

How to cite this article: Rao SS, Ferdinant J, Shikhil P, Ahmed A, Sathya A, Sadacharan D. von Hippel-Lindau Disease with Multi-organ Cysts and Ticking Pheochromocytoma: A Rare Case. World J Endoc Surg 2019;11(1):19-21.

Source of support: No support

Conflict of interest: No conflict of interests declared

\section{CASE}

A 38-year-old hypertensive (since her first pregnancy, 15 years ago) lady presented to the emergency unit with breathlessness of acute onset. On examination, she was found to have a high blood pressure of 210/140 $\mathrm{mm} \mathrm{Hg}$ (hypertensive emergency) and pulmonary edema. She required ventilatory support for 2 days to stabilize her vital parameters. She was diagnosed to have cardiogenic pulmonary edema. Echocardiogram revealed global hypokinesia, suggestive of takotsubo cardiomyopathy. She was started on antiplatelets, furosemide, and other corrective measures. Detailed history revealed palpitation and headache on and off for the last 3 months. Family history suggested that her brother died of cranial space occupying lesion (cerebellar hemangioma) and her mother had history of hypertension and underwent coronary artery bypass surgery (CABG) at 50 years of age. Abdominal examination was unremarkable. Abdominal tomography showed the presence of

(c) The Author(s). 20190pen Access This article is distributed under the terms of the Creative Commons Attribution 4.0 International License (https://creativecommons. org/licenses/by-nc/4.0/), which permits unrestricted use, distribution, and non-commercial reproduction in any medium, provided you give appropriate credit to the original author(s) and the source, provide a link to the Creative Commons license, and indicate if changes were made. The Creative Commons Public Domain Dedication waiver (http://creativecommons.org/publicdomain/zero/1.0/) applies to the data made available in this article, unless otherwise stated. 
multiple pancreatic and renal cysts. Biochemical evaluation revealed higher 24-hour urinary fractionated metanephrines $(150 \mu \mathrm{g} /$ 24 hours, normal range: $24-96)$ and normetanephrines (500 $\mu \mathrm{g} /$ 24 hours, normal range:75-375), suggestive of hypercatecholaminism. The presence of multiple and significant renal cysts bilaterally associated with pancreatic cysts alerted us to look for syndromic association. MR imaging of the abdomen showed a heterogeneous intensity lesion in the left adrenal measuring $4.3 \times 3.7 \mathrm{~cm}$ suggesting the possibility of a PCC (Fig. 1C). It also revealed multiple type I and II Bosniak cysts ( 10 and 11) in both the kidneys, the largest measuring $4.6 \times 4.8 \mathrm{~cm}$, suggesting autosomal dominant polycystic kidney disease. Multiple cysts involving the pancreatic head and tail, the largest measuring $9.8 \times 9.2 \mathrm{~mm}$, were also visualized (Fig. 1A). MRI spine showed a well-defined T1 hypointense and T2 hyperintense oval non-enhancing intramedullary cystic lesion, measuring $2.1 \times 0.8 \mathrm{~cm}$ in the C7-D1 cord with mild cord expansion and edema suggestive of hemangioblastoma (Fig. 1B). MRI brain revealed the presence of a left cerebellar hemangioblastoma (Fig. 1D). I-131 MIBG scan showed an increased uptake in the left suprarenal area suggestive of adrenal PCC (Fig. 2). The presence of PCC, renal, and pancreatic cysts with cerebellar and spinal hemangioblastoma qualifies for the diagnosis of VHL type 2A. PCC tumor excision was contemplated and planned accordingly. She was adequately prepared with alpha blockers (Tab. Prazosin) for a month and beta blockers were added for the last 2 days prior to surgery. She was also advised on high fluid intake
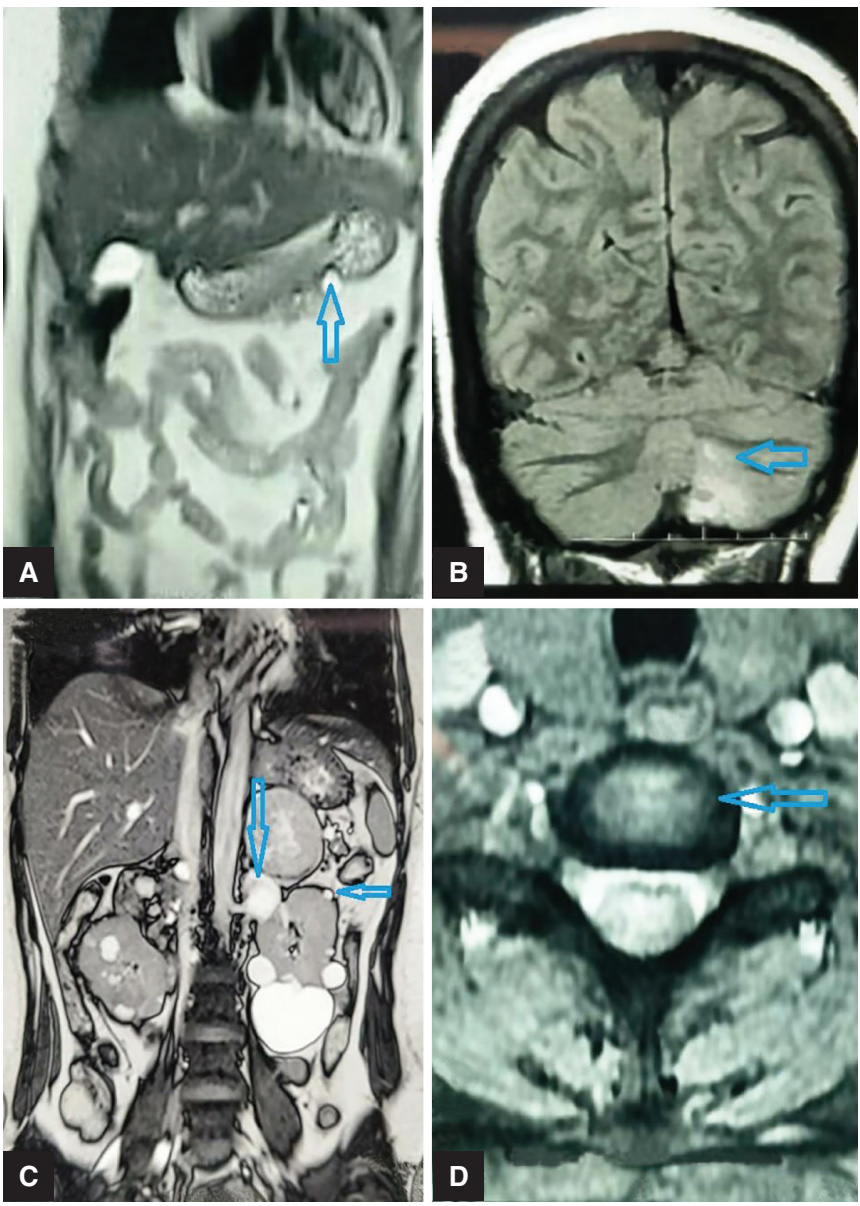

Figs $1 \mathrm{~A}$ to $\mathrm{D}$ : Contrast enhanced computerised tomography pictures: (A) Pancreatic cyst; (B) Cerebellar hemangioblastoma; (C) Left adrenal lesion; (D) Spinal hemangioblastoma

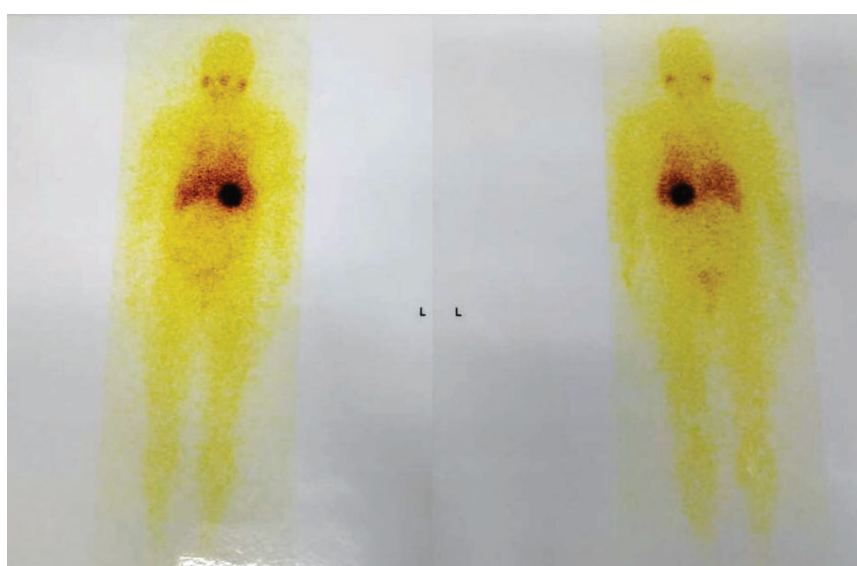

Fig. 2: I-131 MIBG showing uptake in the left suprarenal region

(at least 4 liters per day) and high salt intake for the expansion of the contracted intravascular volume. After adequate preparation, she was taken up for surgery by a laparoscopic technique with three ports. Intraoperatively, visualization of left adrenal tumor became extremely strenuous due to the presence of large renal cysts in the close proximity (Figs $3 \mathrm{~A}$ and $\mathrm{B}$ ). There were multiple renal cysts in the superior pole, the largest measuring $4 \times 5 \mathrm{~cm}$, and in the renal hilar area. These renal cysts appeared separate from the kidney mimicking the adrenal tumor. Also, an extensive dissection in that area would result in inadvertent injury to the kidney, renal hilum, and other surrounding vital structures. The PCC was located just over the left renal hilum. The tumor was dissected all around using
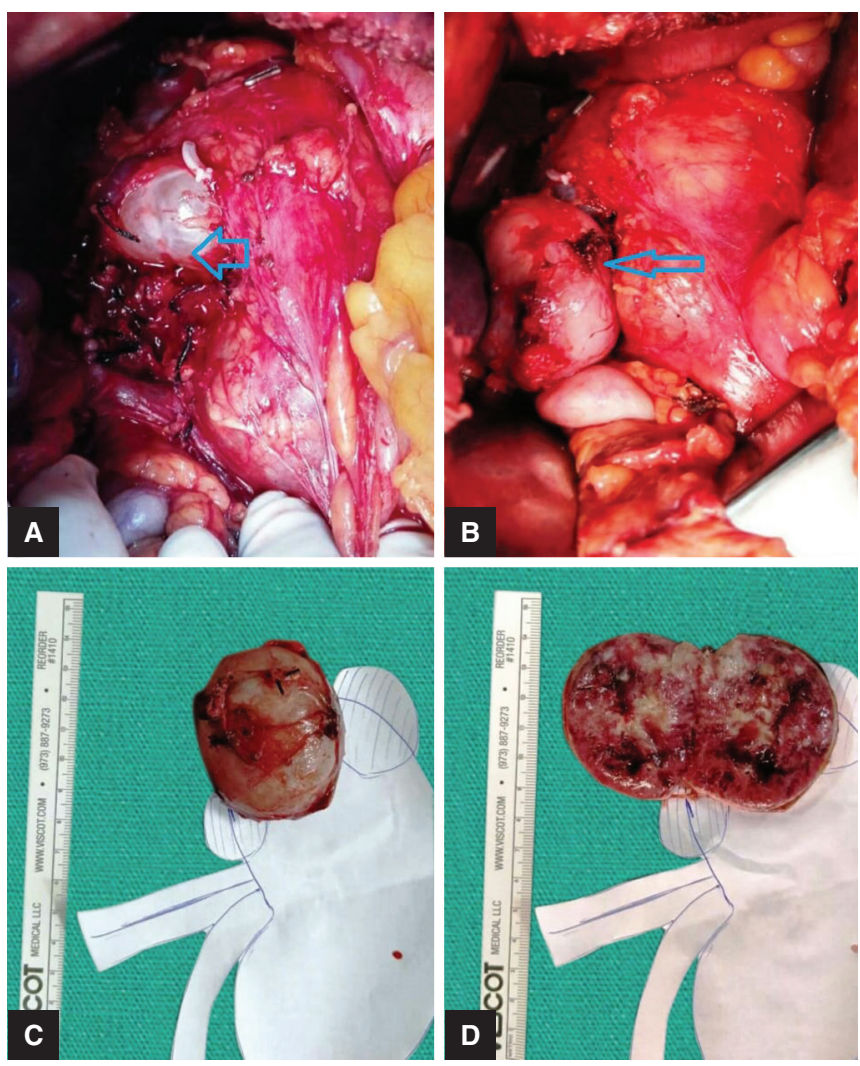

Figs $3 A$ to $D$ : ( $A$ and $B$ ) Intraoperative pictures showing renal cysts and left adrenal lesion; (C) Postoperative gross specimen; (D) Cut surface of the adrenal lesion 
von Hippel-Lindau Disease with Multi-organ Cysts and Ticking Pheochromocytoma

a harmonic scalpel, starting from the superior aspect. The tumor was highly vascular with surface vessels running over it. The renal cysts were located superior and lateral to the tumor. Pancreatic cysts along with the tail of the pancreas were located medially. During inferior dissection of the tumor, close to the renal hilum, extensive neovascularization was present over the tumor. Accidental bleeding occurred and it could not be managed by compression or by clipping. To tackle the tumoral bleed, open surgical approach was undertaken by joining the port sites. After skeletonization of the left renal vein and its drainage into the inferior vena cava, left adrenal vein was ligated. The tumor was dissected all around and excised in toto. During dissection, due care was taken to avoid injuring the surrounding renal and pancreatic cysts and the renal hilar structures. Intraoperatively, hemodynamic fluctuations did occur in spite of prolonged alpha blockade. The maximum blood pressure recorded was 200/110 mm $\mathrm{Hg}$ during tumor handling and the minimum $\mathrm{BP}$ recorded was $80 / 60$ $\mathrm{mm} \mathrm{Hg}$ after the tumor excision. The size of the tumor was $5.7 \times 4.1$ $\times 3.4 \mathrm{~cm}$. The cut surface of the tumor was fleshy with intervening hemorrhagic areas (Figs 3 C and D). The patient was extubated in the operation theatre and postoperatively managed in intensive care unit. She had an uneventful postoperative recovery. Histopathological examination was consistent with adrenal PCC. Her postoperative metanephrines and normetanephrines were normal and blood pressure was under control with a single low-dose antihypertensive.

\section{Discussion}

Our patient had a constellation of multiple renal and pancreatic cysts, PCC, cerebellar, and spinal hemangioblastomas, but absence of retinal angiomas and RCC as described in few other case reports. ${ }^{1}$ Retinal angiomas were not present in our case in spite of cerebellar and spinal hemangioblastomas which is quite uncommon. Ayloo et al. reported a case of VHL in 2016 with retinal angiomas, bilateral RCCs, and multiple pancreatic cysts as opposed to our case. ${ }^{1}$ Our patient also had an acute presentation which led to this syndromic diagnosis. She presented with cardiogenic pulmonary edema due to acute cardiomyopathy of takotsubo type. This type of acute cardiomyopathy is not unusual in PCC and has been reported in $<3 \%$ of patients. ${ }^{8}$ This is a reversible form of cardiomyopathy that recovers completely after an acute attack if managed promptly. This apical ballooning syndrome (takotsubo cardiomyopathy) is thought to occur due to extreme coronary vasospasm by the circulating catecholamines. Our patient has survived both the previous pregnancies, undiagnosed of the disease without any catastrophe. She had undergone two previous caesarean deliveries at 23 and 25 years, respectively, and had no misadventure fortunately. Yaghobi Joybari et al. in 2017 reported a case with unique presentation of well-differentiated invasive pancreatic neuroendocrine carcinoma treated with resection. On follow-up, she was detected with bilateral RCCs and cerebellar hemangioblastomas. ${ }^{2}$ Surgery for cerebellar and spinal hemangioblastomas is a matter of controversy. Our patient does have both the above lesions and is asymptomatic at present. So, a close observation is contemplated for the hemangioblastomas and intervention would be done based on symptomatology or rapid growth. Vaganovs et al. surgically managed cerebellar hemangioblastoma in a young patient with subsequent detection of PCC on follow-up. ${ }^{3}$ The utility of propranolol in delaying the growth of cerebellar hemangiomas in VHL has been described in the literature. ${ }^{3}$ In high-income countries, a majority of VHL are detected on genetic screening and cortical sparing adrenalectomy is an option in bilateral involvements. Medical, psychological, and societal support is generally required in these patients with genetic counseling and communication to the families. ${ }^{4,5}$ Our patient needs lifelong follow-up and genetic screening of her first-degree relatives. PCC is an important component of VHL syndrome and is the second most common cause of death in VHL type $2 \mathrm{~A}$ next to hemangioblastomas. ${ }^{9}$ Our patient presented with PCC with symptoms of headache and hypertension and obtained surgical cure. PCCs can be either adrenal or extra-adrenal and malignancy is not uncommon in VHL. Classical telltale symptoms of PCC might be missing due to low activity. ${ }^{10,11}$ Pancreatic lesions in VHL disease are usually asymptomatic as seen in our case. When symptomatic, they can present with vague symptoms of dyspepsia, diarrhea, and obstructive jaundice. Imaging is an important tool in diagnosing many of these lesions. MRI is the preferred modality for annual surveillance of these adrenal and extra adrenal lesions to look for malignant transformation or appearance of new lesions. ${ }^{12}$

\section{ConCLUSION}

Our patient with VHL type 2A had PCC, bilateral multiple renal cysts, cerebellar and spinal hemangioblastomas, and nonfunctioning pancreatic cysts. The acute presentation of cardiogenic pulmonary edema with takotsubo cardiomyopathy unraveled the diagnosis of $\mathrm{VHL}$, in spite of two earlier uneventful deliveries. Intraoperative identification and dissection of these PCCs is a challenge to the operating surgeon due to the close proximity of the surrounding multiple renal cysts. These patients need a multidisciplinary approach to manage the multiple syndromic components and lifelong follow-up with genetic counseling.

\section{References}

1. Ayloo S, Molinari M. Pancreatic manifestations in von Hippel-Lindau disease: a case report. Int J Surg Case Rep 2016;21:70-72. DOI: 10.1016/j.ijscr.2016.02.031.

2. Yaghobi Joybari A, et al. Von Hippel-Lindau disease. Am J Case Rep 2017;18:1220-1224.

3. Vaganovs P, Bokums K, et al. Von Hippel-Lindau Syndrome: Diagnosis and Management of Hemangioblastoma and Pheochromocytoma. Case Rep Urol 2013;624096:1-4.

4. Shuin T, Yamasaki I, et al. Von Hippel-Lindau Disease: Molecular pathological basis, clinical criteria, genetic testing, clinical features of tumours and treatment. Jpn J Clin Oncol 2006;36(6):337-343. DOI: 10.1093/jjco/hyl052.

5. Dagdeviren Çakir A, et al. Pheochromocytoma Cases as First Manifestion of von Hippel-Lindau. J Clin Res Pediatr Endocrinol 2018;10(2):179-182. DOI: 10.4274/jcrpe.5078.

6. BhargavaS, Rao A, et al. A Case of Von Hippel Lindau Disease. Med J Armed Forces India 2007;63(3):302-304. DOI: 10.1016/S0377-1237(07)80167-5.

7. Perona-Moratalla AB, Serrano-Heras G, et al. Case Report: Efficacy of propranolol in delaying the growth of hemangioblastomas in a Von Hippel Lindau patient. F1000Research 2017;6:256.

8. Chiang YL, Chen PC, et al. Adrenal pheochromocytoma presenting with Takotsubo-pattern cardiomyopathy and acute heart failure: a case report and literature review. Medicine (Baltimore) 2016;95(36):e4846.

9. Rohana AG, Norazmi MK, et al. A Rare Case of Von Hippel Lindau Disease. Med J Malays 2006;61:23-256.

10. Lonser RR, Glenn GM, et al. Von Hippel Landau disease. Lancet 2003; 361:2059-2067. DOI: 10.1016/S0140-6736(03)13643-4.

11. Zhang $L, X u B$, et al. Advanced renal cell carcinoma associated with Von Hippel-Lindau disease: a case report and review of the literature. Oncol Lett 2015;10(2):1087-1090. DOI: 10.3892/ol.2015.3279.

12. Taouli B, Ghouadni M, etal.Spectrum of Abdominal Imaging Findings in von Hippel-Lindau Disease. AJR, Am JRoentgenol, 2003;181:1049-1054. DOI: 10.2214/ajr.181.4.1811049. 